\title{
Theory of Topological Edges and Domain Walls
}

\author{
F. A. Bais, ${ }^{1,2}$ J. K. Slingerland, ${ }^{3,4}$ and S. M. Haaker ${ }^{1}$ \\ ${ }^{1}$ Institute for Theoretical Physics, University of Amsterdam, Valckenierstraat 65, 1018 XE Amsterdam, The Netherlands \\ ${ }^{2}$ Santa Fe Institute, Santa Fe, New Mexico 87501, USA \\ ${ }^{3}$ School of Theoretical Physics, Dublin Institute for Advanced Studies, 10 Burlington Road, Dublin, Ireland \\ ${ }^{4}$ Department of Mathematical Physics, National University of Ireland, Maynooth, Ireland \\ (Received 7 January 2009; revised manuscript received 5 May 2009; published 5 June 2009)
}

\begin{abstract}
We investigate domain walls between topologically ordered phases in two spatial dimensions. We present a method which allows for the determination of the superselection sectors of excitations of such walls and which leads to a unified description of the kinematics of a wall and the two phases to either side of it. This incorporates a description of scattering processes at domain walls which can be applied to questions of transport through walls. In addition to the general formalism, we give representative examples including domain walls between the Abelian and non-Abelian topological phases of Kitaev's honeycomb lattice model in a magnetic field, as well as recently proposed domain walls between spin polarized and unpolarized non-Abelian fractional quantum Hall states at different filling fractions.
\end{abstract}

DOI: 10.1103/PhysRevLett.102.220403

Recently, there has been considerable interest in planar systems which exhibit topological phases. It is of great interest to have a clear understanding of the edges of such systems and of domain walls between regions in different phases. In fractional quantum Hall $(\mathrm{FQH})$ systems, where experimental support for the existence of a variety of topological phases is strongest, observations are almost entirely restricted to edge transport, and proposed devices for probing the topological order rely on interference of tunneling currents between edges [1-3]. In such experiments, the electron density is usually not constant throughout the sample, and islands with different filling fractions form, separated by domain walls. In lattice models with several topological phases, one may induce phase boundaries by varying the local couplings.

We present a method to determine the degrees of freedom of boundaries between topological phases and their relation to the bulk degrees of freedom, based on the condensation of bosonic quasiparticles in auxiliary layered systems. Our method is based on the topological symmetry breaking procedure of Refs. [4-7]. In those papers, only transitions between phases at equal topological central charge were considered-this corresponds to a class of transitions caused by perturbations which do not break parity or time reversal. Here we incorporate transitions which do change the central charge by adding an auxiliary layer to part of the system before any Bose condensation. The central charge is then locally the sum of the central charges of the layers. This allows application in a greater variety of physical settings. We work out two such applications, which involve Kitaev's spin model on the honeycomb lattice [8] and a domain wall between spin polarized and unpolarized non-Abelian FQH liquids [9].

The excitations of topological phases fall into a spectrum topological sectors $a, b, c$, etc., distinguished by topological quantum numbers. One such quantum number
PACS numbers: 05.30.Pr, 11.25.Hf, 73.43.-f, 75.10.Jm

is the topological spin. In $(2+1) \mathrm{D}$, this is a phase factor $\theta_{a}=e^{2 i \pi h_{a}}$, which acts on the wave function when an excitation of type $a$ is rotated by $2 \pi$. The kinematic selection rules for fusion, decay, and scattering of topological excitations are summarized in fusion rules of the form $a \times b \rightarrow \sum_{c} N_{a b}^{c} c$. Here the $N_{a b}^{c}$ are integer coefficients which give the number of independent channels by which $a$ and $b$ may fuse into $c$. Positive real numbers $d_{a}$, the quantum dimensions, give a measure of the number of topological degrees of freedom per $a$ particle. A full description of the bulk of a topological phase requires the structure of a $(2+1) \mathrm{D}$ topological field theory (TQFT), and the boundary of a topological medium can usually be described by a $(1+1)$ D conformal field theory (CFT). Here we will make use only of spins and fusion rules, which are usually the same for bulk and boundary sectors.

One way to match two different phases I and II at a domain wall is to treat them as independent systems without interaction. The wall's sectors are then simply pairs of phase I and phase II sectors. However, this is not always the situation observed in experiments. For example, Camino et al. $[10,11]$ created a setup with $\mathrm{FQH}$ states at filling fractions $\nu=1 / 3$ and $\nu^{\prime}=2 / 5$. The boundary has excitations of charge $e / 15$ and cannot be explained as a simple product of the $\nu=1 / 3$ and $\nu=2 / 5$ boundaries [12]. To describe more general interfaces, we start with two layers in phases I and III, which we allow to partially overlap as indicated in Fig. 1. If we bring the layers close, we may have some binding between degrees of freedom in phases I and III in the overlap region. In particular, a bosonic composite of excitations from the two layers could occur, and, consequently, a condensate of such bosons may form. This condensation will lead to a different phase for the middle region, which we denote by II. If we are given theories $C_{1}$ and $C_{3}$ describing phases I and III, i.e., fusion rules and topological spins for these phases, the topological 
Phase III

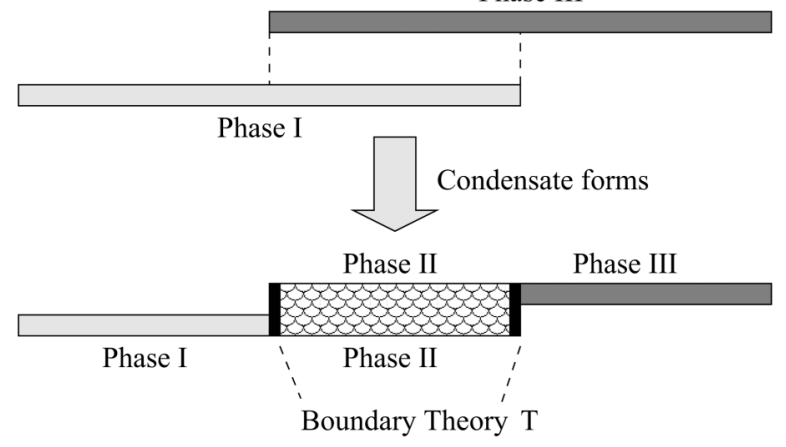

FIG. 1. Side view of two overlapping layers supporting topological phases I and III. If we bring the layers close together, a condensate may form in the overlap region leading to a phase II. The theory $T$ on the left boundary describes excitations that can be divided into bulk excitations of phase I and of phase II and excitations that can propagate only along the boundary. On the right boundary, a similar situation occurs for the same theory $T$, now with III replacing I. The subset of $T$ excitations that are strictly confined to the left and right boundaries is therefore different, in general.

sectors of the layered system will initially be labeled by pairs $\left(a^{\mathrm{I}}, b^{\mathrm{III}}\right)$ of labels from $C_{1}$ and $C_{3}$. We can now proceed as follows.

(i) We find the bosonic sectors. Bosons always have trivial spin, i.e., $\theta_{(a, b)} \equiv \theta_{a} \theta_{b}=1$. Further requirements exist for bosons $a$ with $d_{a}>1$; cf. [7]. (ii) We assume that a condensate of bosonic quasiparticles forms. This causes a change in the topological spectrum and fusion rules. We denote the theory describing the condensed phase by $T$. The spectrum and fusion of $T$ can be found in a way which resembles the branching and fusion analysis for conventional symmetry breaking phase transitions. Sectors of the $C_{1} \otimes C_{3}$ theory branch into $T$ sectors according to branching rules of the form $\left(a^{\mathrm{I}}, b^{\mathrm{III}}\right) \rightarrow \sum_{c} N_{(a, b)}^{c} c^{T}$, where the $N_{(a, b)}^{c}$ are integer branching multiplicities. Some $C_{1} \otimes C_{3}$ sectors may branch to the same $T$ sector and become identified, while others may split into multiple $T$ sectors. In particular, the condensed sectors always branch to the $T$ vacuum, while sectors which are related by fusion with a condensed boson are identified and sectors which are invariant under fusion with a condensed boson split. Branching must commute with fusion, and hence it conserves quantum dimensions. Details and worked examples of the determination of $T$ sectors and fusion are given in Ref. [7]. (iii) While all $T$ sectors have good fusion rules, some do not inherit well defined spin factors from the uncondensed theory, basically because they have nontrivial braiding interaction with a condensed excitation. Excitations from such $T$ sectors pull strings in the condensed medium and are confined. In effect, this means that they are expelled from the bulk and can propagate only on the boundary of the condensed medium. (iv) $T$ sectors which do inherit well defined topological spins from the uncondensed theory survive in the bulk and define a theory $C_{2}$, which describes the fusion and braiding of excitations of phase II.

We now make the crucial observation that, after condensation, excitations in all parts of the system can be labeled by sectors of the $T$ theory. More precisely, the bulk excitations of phase II correspond to unconfined $T$ sectors, while those of phases I and III are labeled as before by pairs $\left(a^{\mathrm{I}}, 1\right)$ and $\left(1, b^{\mathrm{III}}\right)$ of $C_{1} \otimes C_{3}$ labels (with the vacuum label 1 in the layer that cannot be excited in those phases). These pairs correspond to $T$ sectors by the branching rules. This yields unique $T$ sectors (i.e., no splitting) whenever $C_{1}$ and $C_{3}$ do not themselves have bosonic sectors. Boundary excitations correspond to confined $T$ sectors. We can now understand all of the kinematics of processes that may occur when excitations are moved toward or through walls. For example, any $C_{1}$ particle that is identified with a nonconfined $T$ particle can pass through the phase boundary unnoticed and vice versa, while a $C_{1}$ particle that corresponds to a confined $T$ particle cannot enter the region in phase II. Reversely, $T$ particles which are confined in phase II but which can be obtained from a $C_{1}$ sector by branching can pass into the area in phase I after being driven out of phase II. Hence, the true boundary excitations are labeled by confined $T$ sectors which do not correspond to $C_{1}$ sectors. For processes involving three or more excitations, we need to use the fusion rules of $T$. Any process allowed by these rules could, in principle, occur. For example, a $C_{1}$ particle corresponding to a confined $T$ sector $c$ could hit the phase boundary and split into a boundary excitation $a$ and a bulk excitation $b$ of phase II, provided that $c \in a \times b$ according to the fusion rules of $T$. The fusion rules of $T$ are valid throughout. For instance, the fusion channel of two particles in phase I should be preserved even if one of the particles is moved into the region in phase II. The full topological state of the multiphase system should be characterized by specifying the amplitudes for the $T$-fusion channels obtained on successive fusions of all of the quasiparticles that are present. To actually perform the fusions involved, it will usually be necessary to bring the quasiparticles from the bulk regions to the boundary.

There are many applications of these general ideas. For example, coset models in CFT can be analyzed in terms of Bose condensates [7]. The construction of these models by condensation parallels Fig. 1, where phase I is a $G_{k}$ phase, phase III is a $H_{k^{\prime}}$ phase with the opposite chirality, and in the overlap region we obtain a phase with the topological order of the $G_{k} / H_{k^{\prime}}$ coset, after condensation of all available bosons. We continue with two concrete applications of a slightly different, but related, type.

Kitaev's honeycomb model [8] is a model of spins living on the sites of a honeycomb lattice and interacting through nearest neighbor Ising-like interactions. The model is exactly solvable and displays two types of phases [17]: three equivalent gapped Abelian topological phases, with the same topological order as the $\mathbb{Z}_{2}$ toric code and central charge $c=0$, and a gapless phase, which becomes gapped 
TABLE I. Ising and toric code spins and quantum dimensions.

\begin{tabular}{|c|c|c|c|c|c|}
\hline \multicolumn{6}{|l|}{ Ising } \\
\hline$c=1 / 2$ & & 1 & & $\sigma$ & $\psi$ \\
\hline$h_{i}$ & & 0 & & $1 / 16$ & $1 / 2$ \\
\hline$d_{i}$ & & 1 & & $\sqrt{2}$ & 1 \\
\hline \multicolumn{6}{|c|}{$\mathbb{Z}_{2}$ toric code } \\
\hline$c=0$ & 1 & & $e$ & $m$ & em \\
\hline$h_{i}$ & 0 & & 0 & 0 & $1 / 2$ \\
\hline$d_{i}$ & 1 & & 1 & 1 & 1 \\
\hline
\end{tabular}

when a Zeeman term is added to the Hamiltonian and then displays non-Abelian topological order described by the Ising TQFT at $c=1 / 2$. The Abelian phase has four sectors with $\mathbb{Z}_{2} \times \mathbb{Z}_{2}$ fusion rules, and the Ising model has three sectors labeled $1, \sigma$, and $\psi$, with 1 denoting the vacuum and with nontrivial fusion rules given by $\sigma \times \sigma=1+\psi$, $\sigma \times \psi=\sigma$, and $\psi \times \psi=1$.

We wish to consider a situation with an island in the Abelian phase surrounded by a medium in the Ising phase. To achieve this, we take a large disk in the Ising phase (phase I) and place a small disk on top of it, which is in a suitable phase III so that a Bose condensate can form, leaving the bulk of the small disk in the $\mathbb{Z}_{2} \times \mathbb{Z}_{2}$ phase (phase II). The addition of the phase III layer should lower the central charge by $1 / 2$, and so we use an opposite chirality Ising model for phase III. We then take a condensate in the bosonic $(\psi, \psi)$ sector. This example has been worked out in Sec. X of Ref. [7]. Condensation leads to the identifications $(1,1) \sim(\psi, \psi),(\psi, 1) \sim(1, \psi),(\sigma, 1) \sim$ $(\sigma, \psi)$, and $(1, \sigma) \sim(\psi, \sigma)$, while the remaining sector splits: $(\sigma, \sigma)=(\sigma, \sigma)_{1}+(\sigma, \sigma)_{2}$. Hence, the $T$ theory has 6 sectors, and one finds that it has Ising $\times \mathbb{Z}_{2}$ fusion rules. The sectors $(\sigma, 1)$ and $(1, \sigma)$ are confined because they cannot be assigned a consistent spin (the correspond-

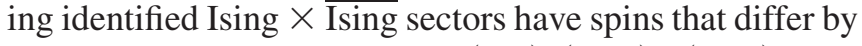
a sign). The unconfined sectors $(1,1),(\sigma, \sigma)_{1},(\sigma, \sigma)_{2}$, and $(\psi, 1)$ correspond precisely to the toric code sectors $1, e$, $m$, and $e m$, respectively, given in Table I.

Let us now look at the wall in between the phases. Of the nontrivial excitations in the interior bulk, the fermionic $(\psi, 1)$ excitation can freely move out through the wall into the exterior region, and the other two bulk excitations cannot. This corresponds well to the results of Ref. [19], where it was shown that free fermionic excitations occur throughout the phase diagram. The confined excitations are expelled from the interior. One, the $(\sigma, 1)$ excitation, can move into the exterior region, while the other, the $(1, \sigma)$ excitation, is strictly confined to the wall. Now consider a $\sigma$ excitation hitting the boundary. From the $T$ theory's fusion rules, we see that $(\sigma, 1)=(1, \sigma) \times(\sigma, \sigma)_{1}=$ $(1, \sigma) \times(\sigma, \sigma)_{2}$. Hence, the $\sigma$ particle can split into a boundary excitation and either an $e$ - or an $m$-type toric code excitation. This corresponds well with the results of Ref. [20], where $\sigma$-like excitations were exhibited in the toric code using superpositions of $e$ - and $m$-type excitations. Pushing another $\sigma$ particle through the phase boundary will allow the confined $(1, \sigma)$ excitations to annihilate, yielding either $(1,1)$ or $(\psi, 1)$. If the two $\sigma$ particles had fusion channel 1 , then the two toric code particles that form will have fusion channel 1 , respectively, $e m \equiv(1, \psi)$, conserving $T$ charge.

Now we turn to the interface between the Moore-Read (MR) Pfaffian FQH state at filling $\nu=1 / 2$ or 5/2 [21] and the non-Abelian spin-singlet (NASS) state of Ardonne and Schoutens at $\nu=4 / 7$ or $18 / 7$ [22]. This was recently considered in Ref. [9]. We will again realize it as a single-layer-two-layer boundary. We concentrate on the non-Abelian parts of the MR and NASS theories here and leave out the $U(1)$ factors (these can be put back in at any point). Consider a disk with $C_{1}=$ Ising, corresponding to $\mathrm{MR}$, with on top of that a smaller disk with $C_{3}=M(4,5)$. The latter CFT is the minimal model with $c=7 / 10$ corresponding to a tricritical Ising model. We give the field content of the Ising and $M(4,5)$ theories in Tables I and II. For the $M(4,5)$ fusion rules, we refer to Ref. [23].

The $\left(\psi, \epsilon^{\prime \prime}\right)$ current is the only bosonic channel in the Ising $\otimes M(4,5)$ model. Table III shows what happens to the various sectors in the model when it condenses. Of the initial 18 sectors, 16 become pairwise identified [because they are equivalent modulo fusion with $\left.\left(\psi, \epsilon^{\prime \prime}\right)\right]$, and the other two split, giving a total of $12 T$ sectors, listed at the top of the table. An analysis along the lines of Ref. [7] shows that the fusion rules of $T$ are given by $T=\mathbb{Z}_{2} \otimes$ $M(4,5)$. The $T$ sectors that are not confined correspond to the sectors of the NASS state; see also Table II. The full $T$ theory does not admit a consistent braid group representation, since the confined sectors cannot be assigned unam-

TABLE II. NASS (phase II) and $M(4,5)$ (phase III).

\begin{tabular}{cccccccccc}
\hline \hline NASS & & 1 & $\sigma_{\uparrow}$ & $\sigma_{\downarrow}$ & $\sigma_{3}$ & $\rho$ & $\psi_{1}$ & $\psi_{2}$ & $\psi_{12}$ \\
\hline$c=6 / 5$ & $h_{i}$ & 0 & $\frac{1}{10}$ & $\frac{1}{10}$ & $\frac{1}{10}$ & $\frac{3}{5}$ & $\frac{1}{2}$ & $\frac{1}{2}$ & $\frac{1}{2}$ \\
& $d_{i}$ & 1 & $(1+\sqrt{5}) / 2$ & $(1+\sqrt{5}) / 2$ & $(1+\sqrt{5}) / 2$ & $(1+\sqrt{5}) / 2$ & 1 & 1 & 1 \\
\hline \hline \hline$M(4,5)$ & & 1 & $\epsilon$ & $\epsilon^{\prime}$ & $\epsilon^{\prime \prime}$ & $\bar{\sigma}$ & $\bar{\sigma}^{\prime}$ \\
\hline$c=7 / 10$ & $h_{i}$ & 0 & $\frac{1}{10}$ & $\frac{3}{5}$ & $\frac{3}{2}$ & $\frac{3}{80}$ & $\frac{7}{16}$ \\
& $d_{i}$ & 1 & $(1+\sqrt{5}) / 2$ & $(1+\sqrt{5}) / 2$ & 1 & $(1+\sqrt{5}) / \sqrt{2}$ & $\sqrt{2}$ \\
\hline \hline
\end{tabular}


TABLE III. Field content of the $T$ theory resulting from a $\left(\psi, \epsilon^{\prime \prime}\right)$ condensate in the Ising $\otimes M(4,5)$ model and governing the kinematics of the NASS and MR states and the domain wall between them. The following rows give the correspondence between $T$ sectors and sectors of the different phases and walls. Note that the quantum dimensions of the $T$ sectors are consistent with all fusion rules and with the decomposition of the MR and $M(4,5)$ sectors. One reads off that the excitations $\bar{\sigma}, \bar{\sigma}^{\prime}$, and $\sigma^{*}$ are strictly confined to the I/II boundary. The same $T$ theory would live on a domain wall between NASS and $M(4,5)$ phases, where $\sigma$ and $\sigma^{*}$ would be strictly confined to the II/III boundary.

\begin{tabular}{|c|c|c|c|c|c|c|c|c|c|c|c|c|}
\hline$T$ theory & 1 & $\sigma_{\uparrow}$ & $\sigma_{\downarrow}$ & $\sigma_{3}$ & $\rho$ & $\psi_{1}$ & $\psi_{2}$ & $\psi_{12}$ & $\sigma$ & $\bar{\sigma}$ & $\bar{\sigma}^{\prime}$ & $\sigma^{*}$ \\
\hline $\begin{array}{l}\text { Corresponding sectors } \\
\text { in Ising } \otimes M(4,5)\end{array}$ & $\begin{array}{l}(1,1) \\
\left(\psi, \epsilon^{\prime \prime}\right)\end{array}$ & $(\sigma, \bar{\sigma}$ & & $\begin{array}{l}(1, \epsilon) \\
\left(\psi, \epsilon^{\prime}\right)\end{array}$ & $\begin{array}{l}\left(1, \epsilon^{\prime}\right) \\
(\psi, \epsilon)\end{array}$ & $(\sigma, \bar{\sigma}$ & & $\begin{array}{l}\left(1, \epsilon^{\prime \prime}\right) \\
(\psi, 1)\end{array}$ & $\begin{array}{l}(\sigma, 1) \\
\left(\sigma, \epsilon^{\prime \prime}\right)\end{array}$ & $\begin{array}{l}(1, \bar{\sigma}) \\
(\psi, \bar{\sigma})\end{array}$ & $\begin{array}{l}\left(1, \bar{\sigma}^{\prime}\right) \\
\left(\psi, \bar{\sigma}^{\prime}\right)\end{array}$ & $\begin{array}{l}(\sigma, \epsilon) \\
\left(\sigma, \epsilon^{\prime}\right)\end{array}$ \\
\hline$d_{i}$ & 1 & $(1+\sqrt{5}) / 2$ & $(1+\sqrt{5}) / 2$ & $(1+\sqrt{5}) / 2$ & $(1+\sqrt{5}) / 2$ & 1 & 1 & 1 & $\sqrt{2}$ & $(1+\sqrt{5}) / \sqrt{2}$ & $\sqrt{2}$ & $(1+\sqrt{5}) / \sqrt{2}$ \\
\hline Phase I: MR & 1 & & & & & & & $\psi$ & $\sigma$ & & & \\
\hline Phase II: NASS & 1 & $\sigma_{\uparrow}$ & $\sigma_{\downarrow}$ & $\sigma_{3}$ & $\rho$ & $\psi_{1}$ & $\psi_{2}$ & $\psi_{12}$ & & $\overline{\bar{\sigma}}$ & $\bar{\sigma}^{\prime}$ & $\sigma^{*}$ \\
\hline Phase III: $M(4,5)$ & 1 & & & $\epsilon$ & $\epsilon^{\prime}$ & & & $\epsilon^{\prime \prime}$ & & $\bar{\sigma}$ & $\bar{\sigma}^{\prime}$ & \\
\hline Confined on II/III wall & & & & & & & & & $\sigma$ & & & $\sigma^{*}$ \\
\hline
\end{tabular}

biguous spins. We recall (cf. [6]) that this is no problem, since the full $T$ theory has only a strictly one-dimensional interpretation.

In Table III, we have also indicated which $T$ sectors correspond to excitations in the various planar regions and which are strictly confined to particular walls. More precisely, we give the $T$ sectors that extend from the I/II wall into the exterior MR (phase I) region in the fourth row and the sectors that extend from the wall into the interior NASS (phase II) region in the fifth row. Excitations in the three remaining sectors occur only on the I/II wall. The $\psi_{12}$ sector of the NASS phase is identified with the MR-sector $\psi$, which means that the $\psi$ or $\psi_{12}$ excitations can propagate right through the wall. Again, the fusion rules of the $T$ theory fix the kinematically allowed channels by which particles which hit the wall can split. For instance, from the $T$ fusion rule $\sigma \times \bar{\sigma}=\sigma_{\uparrow}+\sigma_{\downarrow}$, we find that a $\sigma_{\uparrow}$ coming from the interior region can split into a $\sigma$ going into the $\mathrm{MR}$ region and a $\bar{\sigma}$ staying in the wall. However, since $\bar{\sigma} \times$ $\sigma^{*}=\sigma_{\uparrow}+\sigma_{\downarrow}+\psi_{1}+\psi_{2}$, the $\sigma_{\uparrow}$ excitation may instead split into two wall excitations $\bar{\sigma}$ and $\sigma^{*}$. This scenario may also be turned around; two strict boundary excitations may fuse into a state that is not confined. Obviously, there are many more possible processes, and we refrain from listing them all here.

A final comment concerns the relaxation of qubits near a wall [24]. If we encode a topological qubit in the NASS phase, for example, in the fusion channel of a pair of excitations, the qubit may relax to the lowest energy state by transferring a neutral excitation to the boundary. For example, for pairs of $\sigma$-type excitations, we have the fusion rules $\sigma_{3} \times \sigma_{3}=1+\rho$ and $\sigma_{\downarrow} \times \sigma_{\uparrow}=\psi_{12}+\sigma_{3}$, so these pairs can relax under emission of a $\rho$ excitation. A $\rho$ excitation may convert into one of the pairs $\left(\sigma^{*}, \sigma^{*}\right)$, $(\bar{\sigma}, \bar{\sigma})$, or $\left(\bar{\sigma}, \bar{\sigma}^{\prime}\right)$, which are all strictly confined to the interface. Alternatively, we may have $\rho \rightarrow\left(\sigma^{*}, \sigma\right)$, where $\sigma^{*}$ is confined to the wall but $\sigma$ can enter the MR region.

In conclusion, one may describe phase separated topological phase media using auxiliary layers and Bose condensation. An important question is how to fix the appropriate auxiliary theory when the jump in central charge between phases exceeds 1 . It would also be of interest to study how our results relate to those of Gils et al. [25].

We thank Professor K. Schoutens for useful discussions.

[1] S. Das Sarma et al., Phys. Rev. Lett. 94, 166802 (2005).

[2] C. Nayak et al., Rev. Mod. Phys. 80, 1083 (2008).

[3] P. Bonderson et al., Ann. Phys. (N.Y.) 323, 2709 (2008).

[4] F. A. Bais et al., Phys. Rev. Lett. 89, 181601 (2002).

[5] F. A. Bais et al., J. High Energy Phys. 05 (2003) 068.

[6] C. J. M. Mathy and F. A. Bais, Ann. Phys. (N.Y.) 322, 709 (2007).

[7] F. A. Bais and J. K. Slingerland, Phys. Rev. B 79, 045316 (2009).

[8] A. Kitaev, Ann. Phys. (N.Y.) 321, 2 (2006).

[9] E. Grosfeld and K. Schoutens, arXiv:0810.1955.

[10] F. E. Camino et al., Phys. Rev. B 72, 075342 (2005).

[11] F. E. Camino et al., Phys. Rev. Lett. 95, 246802 (2005).

[12] We will deal with this situation and with more general hierarchy states [13-15] in a separate publication [16].

[13] F. D. M. Haldane, Phys. Rev. Lett. 51, 605 (1983).

[14] B. I. Halperin, Phys. Rev. Lett. 52, 1583 (1984).

[15] P. Bonderson and J. K. Slingerland, Phys. Rev. B 78, 125323 (2008).

[16] F. A. Bais et al. (unpublished).

[17] In extensions of the model, a third type of gapped phase has been found [18].

[18] C. Nash and D. O'Connor, Phys. Rev. Lett. 102, 147203 (2009).

[19] G. Kells et al., Phys. Rev. Lett. 101, 240404 (2008).

[20] J. R. Wootton et al., Phys. Rev. B 78, 161102 (2008).

[21] G. Moore and N. Read, Nucl. Phys. B360, 362 (1991).

[22] E. Ardonne and K. Schoutens, Phys. Rev. Lett. 82, 5096 (1999).

[23] F. Di Francesco et al., Conformal Field Theory (Springer, New York, 1997).

[24] R. Ilan et al., arXiv:0803.1542 [Phys. Rev. B (to be published)].

[25] R. Gils et al., arXiv:0810.2277. 